\author{
DOI 10.15593/24111678/2016.01.04 \\ УДК 656.073 .4
}

\author{
Н.В. Лобов, С.А. Пестриков, С.А. Монченко \\ Пермский национальный исследовательский \\ политехнический университет, Пермь, Россия
}

\author{
ОЦЕНКА ЭКОНОМИЧЕСКОЙ ЭФФЕКТИВНОСТИ \\ ПРИМЕНЕНИЯ ВЗВЕШИВАЮЩЕГО УСТРОЙСТВА, \\ ПОЗВОЛЯЮЩЕГО КОРРЕКТИРОВАТЬ НОРМЫ \\ РАСХОДА ТОПЛИВА ДЛЯ СПЕЦТЕХНИКИ, \\ НА ПРИМЕРЕ МУСОРОВОЗА МК-20
}

\begin{abstract}
Рассматривается методика корректировки нормативов расхода топлива для спецтехники на примере мусоровоза МК-20. Обосновывается актуальность проблемы и необходимость корректировки норм расхода топлива. Приводится описание взвешивающего устройства, позволяющего оценивать массу твердых бытовых отходов по величине давления рабочей жидкости в наиболее нагруженном гидроцилиндре мусоровоза. Рассматривается порядок расчета существующей методики определения норм расхода топлива на спецтехнике. Указываются недостатки существующей методики, связанные со спецификой работы мусоровоза, которая проявляется в постепенной загрузке бункера мусоровоза отходами при прохождении маршрута. Предлагается дифференцировать расчет норм расхода топлива на отдельные участки между пунктами сбора отходов. При проведении исследования были соблюдены необходимые допущения. Приводится расчет одного маршрута мусоровоза МК-20, состоящего из двух рейсов. При анализе полученных результатов предлагается уменьшить значение надбавки к общему расходу топлива на выполнение транспортной работы по перевозке технологического груза. Проводится экономическая оценка эффективности методики. Отмечаются положительные факторы, увеличивающие эффективность, и описываются отрицательные последствия, утяжеляющие эффект. Рассчитывается срок окупаемости инвестиций при установке системы ИМО-1 на мусоровоз МК-20. Рассматривается возможность применения данной методики на предприятиях. В качестве примера дается описание необходимых корректировок в программном обеспечении «RG-SOFT: Вывоз мусора». Представлено дальнейшее направление развития данной работы. Основываясь на полученных результатах, можно рекомендовать данную систему предприятиям, занимающимся сбором и вывозом твердых бытовых отходов, и проводящим грамотную ресурсосберегающую политику.

Ключевые слова: нормирование расхода топлива, спецтехника, мусоровоз, система определения массы отходов, экономическая эффективность, срок окупаемости.
\end{abstract}




\author{
N.V. Lobov, S.A. Pestrikov, S.A. Monchenko
}

Perm National Research Polytechnic University, Perm, Russian Federation

\title{
ASSESSMENT OF ECONOMIC EFFICIENCY OF USE OF THE WEIGHER ALLOWING TO CORRECT RATES OF FUEL SPECIAL MACHINERIES FOR EXAMPLE GARBAGE TRUCK MK-20
}

\begin{abstract}
The technique of adjusting fuel standards for example garbage truck MK-20. Describes the importance of the problem and the need to adjust fuel consumption rates. A description of the weighing device, which allows to estimate the mass of municipal solid waste largest hydraulic fluid pressure in the most loaded garbage truck hydraulic cylinder. We consider the procedure for the calculation of the current methodology for determining the fuel consumption standards on special machineries. In this paper we identified shortcomings of the existing methods, related to the specific work of a garbage truck, which is manifested in the progressive loading garbage truck waste bunker during the passage of the route. The article suggested differentiated calculation of fuel consumption standards in separater areas between the points of waste collection. In conducting the study have been met necessary assumptions. The article provides the calculation of the garbage truck route MK-20, consisting of two flights. In the analysis of the results proposed to reduce the value of the allowance to the total fuel consumption in the transport performance of the work on the transport of cargo. Through economic performance evaluation techniques. Highlighted positive factors, increasing efficiency, and describes the negative effects, the effect of making heavier. Calculate the payback period of investment in the installation system IMO-1 on garbage truck MK-20. As an example, a description of the necessary adjustments in the software "RG-SOFT: "Garbage removal". The possibility of using this technique in the workplace. Presented by the future direction of this work. Based on these results, we can recommend this system to enterprises engaged in the collection and disposal of solid waste, and conducting a competent resource-saving policy.
\end{abstract}

Keywords: fuel consumption rationing, special machineries, garbage truck, a system for determining the mass of waste, economic efficiency, payback period.

Основная задача нормирования - обеспечить применение при планировании и в производстве технически и экономически обоснованных прогрессивных норм расхода топлива в целях более эффективного его использования, рационального распределения и обеспечения экономии. Применение объективных скорректированных норм позволит экономить топливо, прежде всего за счет устранения возможных причин его перерасхода: хищение, неудовлетворительное техническое состояние автомобиля, неквалифицированное вождение.

Методические рекомендации, порядок применения, формулы и методы расчета нормативного расхода топлив для спецтехники представлены в Постановлении Госстроя России от 09.03.2004 № 36 «Об утверждении Рекомендаций по расходу топлива машинами для содержания, ремонта автомобильных дорог и объектов внешнего благоустройства поселений». Однако в данном документе приведена общая формула расчета, которая не учитывает специфику работы мусоровоза, в частности его постепенную загрузку. 
В результате анализа данного вопроса нами была предложена методика корректировки норм расхода топлива в зависимости от степени загрузки мусоровоза. Оценить массу ТБО в бункере мусоровоза стало возможно с использованием системы ИМО-1.

Определение веса поднимаемого груза осуществляется по величине давления рабочей жидкости в наиболее нагруженном гидроцилиндре мусоровоза $[1,2]$. Во время подъема бака фиксируются давление и температура рабочей жидкости, прибор ИМО-1 сохраняет и анализирует полученные данные. Собранная информация сохраняется в памяти прибора и передается на сервер при помощи пакетной передачи данных через радиоинтерфейс (GPRS). Внешний вид устройства представлен на рис. 1.
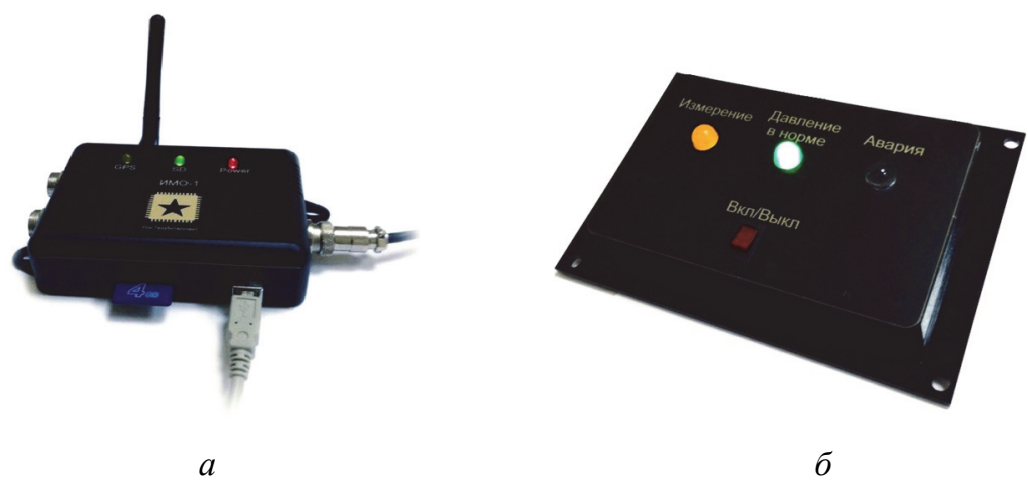

Рис. 1. Внешний вид устройства: $a-$ ИМО-1; $\sigma$ - выносной светодиодный индикатор

Устройство ИМО-1 применяется для работы на подвижной технике, оснащенной погрузочно-разгрузочным оборудованием с гидроприводом, и предназначено [3]:

- для предотвращения подъема груза, вес которого превышает предельно допустимый для погрузочно-разгрузочного оборудования;

- сбора и передачи пользователю данных о маршруте движения техники, местах погрузочно-разгрузочных работ и весе груза, при каждом подъеме;

- оповещения оператора световым и звуковым сигналом об аварийной ситуации;

- дистанционного контроля работы гидравлического оборудования. 
Работа машин производится в различных климатических, дорожно-транспортных и иных эксплуатационных условиях, в связи с чем в данном документе предусмотрена система надбавок, корректирующих представленные удельные показатели расхода топлива [4]. Надбавки даны в процентах от общего расхода топлива для конкретной машины за время ее работы в течение заданного времени (смена, сутки и т.п.). Суммарная относительная надбавка к расходу топлива исчисляется в долях единицы и определяется по формуле

$$
Д=Д_{1}+Д_{2}+Д_{3}+Д_{4}+Д_{5}+Д_{6},
$$

где Д 1 - надбавка при работе спецмашин в черте города с частыми остановками - до $10 \%$; Д 2 - надбавка на работу спецмашин в зимний период; Д - надбавка для автомобилей, находящихся в длительной эксплуатации; Д 4 - надбавка при работе в городах с большой плотностью населения; Д - надбавка к общему расходу топлива на выполнение транспортной работы по перевозке технологического груза - до $10 \%$; Д $_{6}$ - надбавка к общему расходу топлива для машин циклического действия, удаляющих собранные отходы и загрязнения на свалки.

Удельный расход топлива для специальных машин, имеющих автомобильные или факторные шасси и оснащенных навесным оборудованием, привод которого осуществляется от двигателя шасси, определяется по формуле

$$
Q=0,01 \cdot\left[H_{s} \cdot\left(S-S_{1}\right)+Q_{1} \cdot S_{1}\right] \cdot(1+\text { Д }),
$$

где $Q$ - общий расход топлива; $H_{s}$ - линейный расход топлива, л/100 км; $S$ - общий пробег машины, км; $S_{1}$ - пробег машины при работе спецоборудования, км; Д - суммарная относительная надбавка к линейному расходу топлива; $Q_{1}$ - расход топлива при работе спецоборудования, л/ч, л/100 км, л/цикл.

Для корректировки и более точного расчета расхода топлива мусоровозов предложено учитывать постепенное увеличение значения

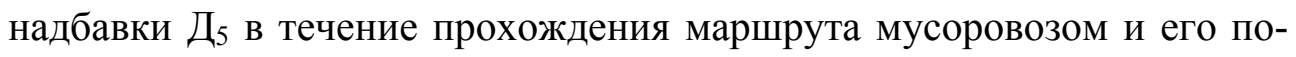
степенной загрузки.

В качестве примера был выбран мусоровоз МК-20 [5, 6], совершающий сбор и вывоз ТБО в Мотовилихинском районе г. Перми. Данный мусоровоз за один день совершает два рейса. Маршрут мусоровоза не меняется, вывоз ТБО производится каждый день $[7,8]$. 
По предложенной методике общая формула расчета расхода будет равна сумме расходов топлива за каждый участок маршрута между местами сбора ТБО. При этом в формуле для каждого последующего участка надбавка Д

При первом рейсе мусоровоз осуществляет сбор ТБО с 6 контейнерных площадок, при втором - с 7. Мусоровоз начинает маршрут от стоянки производственной фирмы ООО «Пламя», расположенной по адресу: ул. Соликамская, 281. После сбора ТБО мусоровоз отправляется на место уплотнения отходов, находящееся на территории ООО «Пламя».

Стоить отметить, что данное исследование и расчет являются теоретическими и направлены на получение первичных результатов. При расчете был принят ряд допущений:

1. Маршрут мусоровоза каждый день остается неизменным.

2. Количество и порядок прохождения мест сбора ТБО остается неизменным.

3. Количество ТБО распределено между контейнерными площадками равномерно.

4. Сбор и вывоз ТБО с мест их накопления осуществляется ежедневно.

Расчет участков маршрута первого и второго рейсов представлен в табл. 1 и 2 соответственно.

Таблица 1

Результаты расчета первого рейса

\begin{tabular}{|c|c|c|c|}
\hline Номер участка & Пробег, км & $Д_{5}$ & Расход $Q$, л \\
\hline 1 & 3,14 & 0 & 1,202527 \\
\hline 2 & 1,23 & 1,66 & 0,491842 \\
\hline 3 & 11,89 & 3,32 & 4,613223 \\
\hline 4 & 1,66 & 4,98 & 0,673474 \\
\hline 5 & 1,44 & 6,64 & 0,595215 \\
\hline 6 & 0,82 & 8,3 & 0,354038 \\
\hline 7 & 9,86 & 10 & 4,037105 \\
\hline Итого & 30,04 & & 11,96743 \\
\hline
\end{tabular}

Таблица 2

Результаты расчета второго рейса

\begin{tabular}{|c|c|c|c|}
\hline Номер участка & Пробег, км & Д $_{5}$ & Расход $Q$, л \\
\hline 1 & 9,89 & 0 & 3,734934 \\
\hline 2 & 1 & 1,42 & 0,400534 \\
\hline 3 & 0,35 & 2,84 & 0,1553 \\
\hline 4 & 0,85 & 4,26 & 0,351563 \\
\hline
\end{tabular}


Окончание табл. 2

\begin{tabular}{|c|c|c|c|}
\hline Номер участка & Пробег, км & Д $_{5}$ & Расход $Q$, л \\
\hline 5 & 0,11 & 5,68 & 0,06448 \\
\hline 6 & 1,41 & 7,1 & 0,582379 \\
\hline 7 & 0,51 & 8,52 & 0,226844 \\
\hline 8 & 10,7 & 10 & 4,375768 \\
\hline Итого & 24,82 & & 9,891802 \\
\hline
\end{tabular}

В результате расчета и корректировки надбавки Д 5 была получена зависимость степени загрузки мусоровоза от его пробега между площадками (рис. 2).

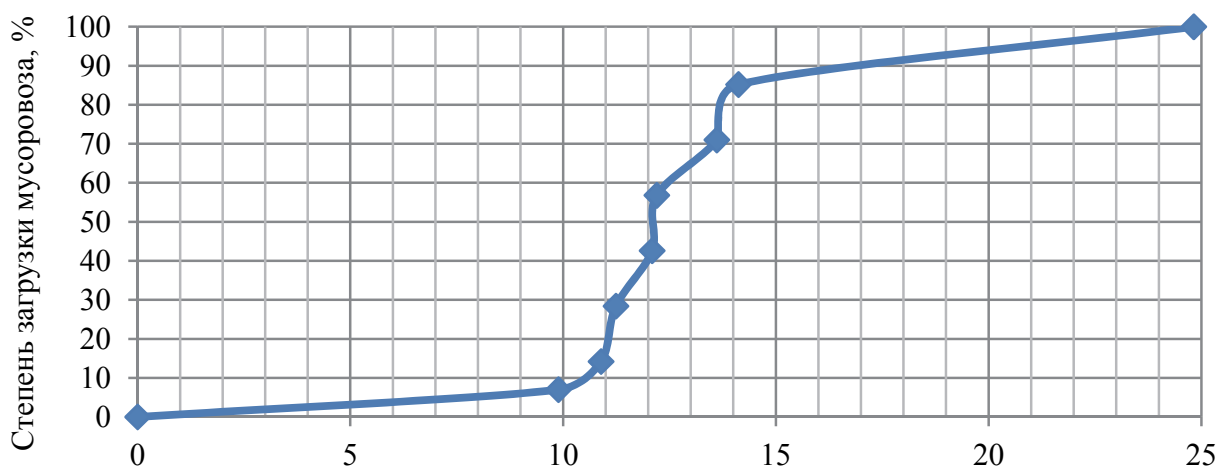

Пробег, км

Рис. 2. Зависимость степени загрузки от пробега для второго рейса

В результате анализа полученных результатов было принято при расчете норм расходов топлива для мусоровозов снизить значение надбавки Д 5 до 5 \%. Данное значение надбавки позволяет приблизить общий расход к сумме расходов на каждом участке маршрута мусоровоза. Таким образом, при расчете, например, расхода для первого рейса с помощью существующей методики, но с надбавкой Д $5=5 \%$, расход топлива будет равным 11,93 л, а при расчете, предложенном дискретным методом, $-11,96$ л. Это дает возможность экономить топливо на стадии планирования, что позволит решить проблемы, перечисленные выше.

Также важным моментом разработки и внедрения методики корректировки норм расхода топлива для спецтехники является количественная оценка показателей экономической эффективности $[9,10]$. Для экономической эффективности данным показателем будет являться 
срок окупаемости капитальных вложений, затраченных на разработку методики и установку системы определения массы ТБО. Оценка производится для системы ИМО-1, установленной на мусоровозе КО-415.

Для дальнейшей оценки определим факторы, повышающие эффективность, а также отметим отрицательные последствия, утяжеляющие эффект [11].

К первой группе можно отнести следующие факторы:

- уменьшение отказов за счет предупреждения перегруза мусоровоза;

- назначение действительных тарифов на вывоз ТБО;

- уменьшение расхода топлива за счет определения нормативов;

- повышение эффективности управления процессом;

- анализ состояния степени загруженности контейнерных площадок.

К отрицательным факторам можно отнести следующие:

- затраты на сбор и установку;

- потребность в квалифицированном сотруднике;

- внедрение в конструкцию (необходимость переоснащения).

На данный момент из всех перечисленных факторов наиболее объективную количественную оценку можно дать лишь двум: уменьшение расхода топлива за счет определения нормативов и затраты на сбор и установку системы ИМО-1.

Исходя из расчетов данной работы, общий расход топлива мусоровоза МК-20 за один день при существующей методике равен 22,674 л. При разработанной методике с учетом корректировки общий расход равен 21,859 л.

При сравнении двух вариантов расчет норм расхода становится очевидным, что расчет при помощи разработанной методики более экономичен. Данный вариант позволяет экономить 0,859 л топлива ежедневно. С учетом того что вывоз ТБО должен осуществляться ежедневно, месячная экономия топлива в среднем составит 24,45 л, что в денежном эквиваленте равняется 820 руб.

Стоимость установки системы ИМО-1 на мусоровоз КО-415 с учетом стоимости оборудования и материалов составляет порядка 25 тыс. руб. Исходя из этого, рассчитаем срок окупаемости инвестиций в данный проект. 
Срок окупаемости - это минимальный временной интервал (от начала осуществления проекта), за пределами которого интегральный эффект становится и остается в дальнейшем положительным [12]. Иными словами, это период времени, начиная с которого первоначальные вложения и другие затраты, связанные с инвестиционным проектом, покрываются суммарными результатами его осуществления. Срок окупаемости определим без использования метода дисконтирования. За шаг расчета принимаем один квартал. Количество шагов принимаем равным 15, т.е. горизонт расчета составит 5 лет.

Недисконтированный срок окупаемости является величиной, обеспечивающей равенство в формуле

$$
\sum_{t=1}^{T}\left(P_{t}-3_{t}-K_{t}\right)=0
$$

На основе расчетов построен график окупаемости инвестиций, который приведен на рис. 3.

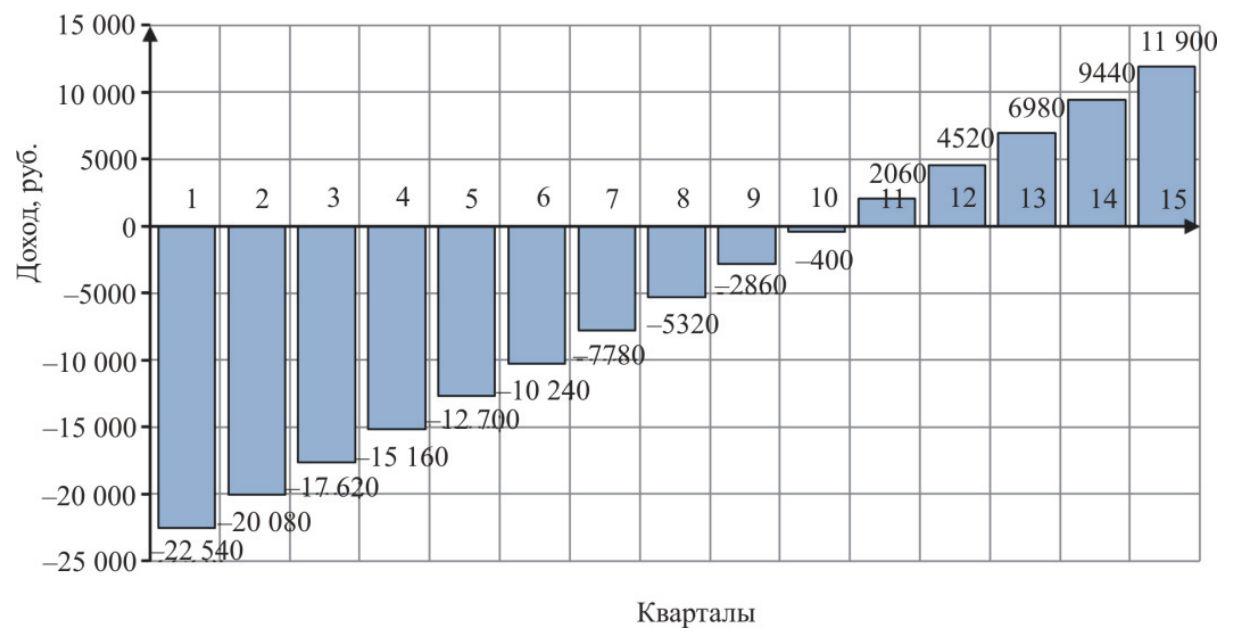

Рис. 3. График окупаемости инвестиций

Согласно графику срок окупаемости инвестиций составит 10 кварталов. Таким образом, система ИМО-1 окупится за 30 месяцев (2,5 года). При этом учтен лишь один фактор снижения расхода топлива.

Дальнейшее практическое исследование и постановка эксперимента по определению массы ТБО с помощью системы ИМО-1 позволят более точно определить расход топлива на каждом участке мар- 
шрута мусоровоза. Для этого необходимо будет увеличить объем выборки и принять во внимание следующие факторы формирования количества ТБО:

- в понедельник наблюдается увеличение количества ТБО, связанное с большим потреблением продуктов в выходные дни;

- по этой же причине увеличивается количество ТБО после праздничных и нерабочих дней;

- в зимний период наблюдается увеличение количества ТБО, что связано с изменением рациона питания и уменьшением времени нахождения жильцов в своих домах.

Усовершенствованная методика корректирования норм расхода топлива позволяет рассчитывать нормы расхода топлива для мусоровозов на любом участке его маршрута. При применении данной методики образуется не условная, а реальная, фактическая экономия. В данном случае экономия появляется только при строгом поддержании исправного состояния мусоровоза и квалифицированном вождении. Внедрение позволяет достичь экономического эффекта за счет снижения затрат на приобретение топлива.

Для простоты применения на любом предприятии, использующем спецтехнику и нормирующем расход топлива, предлагается внести необходимые изменения в используемую программу $[13,14]$. Поскольку маршрут мусоровоза на каждый день определен, известен пробег до каждой контейнерной площадки и с помощью системы ИМО-1 появится возможность определения массы ТБО на каждой площадке. Все это позволит более точно рассчитывать норму расхода топлива путем корректирования надбавки $Д_{5}$.

В качестве примера применения данной методики рассмотрим программу, основанную на языке C++, - «RG-SOFT: Вывоз мусора» [15]. Данная программа служит для облегчения необходимых расчетов работы предприятия, связанной со сбором и вывозом отходов, в том числе определение норм расхода топлива. Пример рабочего окна программы представлен на рис. 4.

В таблице, представленной на рис. 4, при использовании методики корректировки норм расхода топлива появятся колонки пробега между каждыми площадкам и массы ТБО в бункере. Нормируемые и фактические расходы ГСМ будут представлены как суммы расходов за каждую часть маршрута. 




Рис. 4. Рабочее окно программы «RG-SOFT: Вывоз мусора»

На практике данная система позволит осуществлять экономию не только за счет уменьшения расхода топлива. Остальные факторы также оказывают существенное влияние на эффективность эксплуатации спецтехники и, следовательно, имеют высокий ресурсосберегающий потенциал, однако их количественная оценка в настоящее время затруднена. Для приемлемой оценки этих факторов необходимо провести исследования эффектов от внедрения данной системы в условиях реальных предприятий.

В результате проведенного исследования были сформулированы следующие выводы:

1. Существующие методики расчета норм расхода топлива для спецтехники не учитывают в полной мере специфику работы мусоровоза и не позволяют обоснованно определить точные нормативы.

2. Система определения массы ТБО позволит корректировать нормы расхода топлива в зависимости от загруженности мусоровоза.

3. В результате теоретического исследования рекомендовано снизить значение надбавки Д5 для мусоровозов с 10 до $5 \%$.

4. Установка системы ИМО-1 и внедрение корректировки норм расхода топлива позволит экономить до 820 руб. в месяц. Срок окупаемости при этом составит 2,5 года. Однако при дальнейшем учете остальных положительных факторов системы ИМО-1 срок окупаемости может уменьшиться. 
5. Данная методика корректировки может применяться на предприятиях, занимающихся сбором и вывозом ТБО, с небольшими изменениями в программном обеспечении.

\section{Список литературы}

1. Лобов Н.В., Мальцев Д.В., Генсон Е.М. Система контроля веса отходов, загружаемых манипулятором мусоровоза в местах их сбора // Модернизация и научные исследования в транспортном комплексе: материалы междунар. науч.-практ. конф. - Пермь: Изд-во Перм. нац. исслед. политехн. ун-та, 2012. - Т. 1. - С. 145-146.

2. Аналитическое обоснование возможности определения массы твердых бытовых отходов, загружаемых коммунальным транспортом в местах сбора / Н.В. Лобов, Р.Н. Хмелев, Д.В. Мальцев, Е.М. Генсон // Строительные и дорожные машины. - 2012. - № 7. - С. 6-8.

3. Лобов Н.В., Фомина Н.И., Мальцев Д.В. Выбор рационального способа определения массы перевозимого груза мусоровозным транспортом. // Вестник Пермского государственного технического университета. Охрана окружающей среды, транспорт, безопасность жизнедеятельности. - Пермь, 2011. - № 1. - С. 119-123.

4. Копотилов В.И. Автомобили: Теоретические основы / ТюмГНГУ. - Тюмень, 1999. - 403 с.

5. Мусоровоз МК-20. Руководство по эксплуатации / OAO «Саранский завод автосамосвалов». - Саранск, 2008. - 55 с.

6. Кузнецов В.М. Коммунальная техника 2001. - М.: РИА «Россбизнес», 2001. - $176 \mathrm{c.}$

7. Твердые бытовые отходы (сбор, транспорт и обезвреживание): справ. / В.Г. Систер [и др.]. - М.: Изд-во Акад. коммун. хоз-ва им. К.Д. Памфилова, 2001. - 319 с.

8. Концепция обращения с твердыми бытовыми отходами в Российской Федерации // Чистый город. - 2003. - № 2(22). - С. 36-48.

9. Карбанович И.И. Экономия автомобильного топлива: опыт и проблемы. - М.: Транспорт, 1992. - 145 с.

10. Ерохов В. И. Экономичная эксплуатация автомобиля. - М.: Изд-во ДОСААФ, 1986. - 126 с.

11. Лавриков И.Н., Пеньшин Н.В. Экономика автомобильного транспорта: учеб. пособие. - Тамбов: Изд-во ТГТУ, 2011. - 116 с.

12. Сичинава Г.А. Оценка экономической эффективности инвестиционных проектов в строительстве: метод. указания к экономической части дипломных проектов / УГТУ. - Ухта, 2010. - 98 с. 
13. Туревский И.С. Экономика и управление автотранспортным предприятием. - М.: Высшая школа, 2005. - 222 с.

14. Гловацкий Л.В., Гасанов П.Г., Васильев С.В. Управление отходами: вчера, сегодня, завтра [Электронный документ] // Справочноинформационная система «Отходы.ру». - 2008. - URL: www.waste.ru/modules/section/item.php?itemid=15 (дата обращения: 26.01.2016).

15. Садохина Е.Л. Программное обеспечение в экологии. Существующее положение и перспектива [Электронный документ] // Справочно-информационная система «Отходы.ру». - 2006. - URL: www.waste.ru/modules/section/item.php?itemid=63 (дата обращения: 26.01.2016).

\section{References}

1. Lobov N.V., Mal'tsev D.V., Genson E.M. Sistema kontrolia vesa otkhodov, zagruzhaemykh manipuliatorom musorovoza $\mathrm{v}$ mestakh ikh sbora [The monitoring system weight of the waste loaded arm garbage truck in their places of gathering]. Materialy mezhdunarodnoi nauchno-prakticheskoi konferentsii "Modernizatsiia i nauchnye issledovaniia $v$ transportnom komplekse”. Permskii natsional'nyi issledovatel'skii politekhnicheskii universitet, 2012, vol. 1, pp. 145-146.

2. Lobov N.V., Khmelev R.N., Mal'tsev D.V., Genson E.M. Analiticheskoe obosnovanie vozmozhnosti opredeleniia massy tverdykh bytovykh otkhodov, zagruzhaemykh kommunal'nym transportom $\mathrm{v}$ mestakh sbora [Analytical justification of the possibility of determining the mass of solid waste, municipal transport loaded gathering places]. Stroitel'nye i dorozhnye mashiny, 2012, no. 7, pp. 6-8.

3. Lobov N.V., Fomina N.I., Mal'tsev D.V. Vybor ratsional'nogo sposoba opredeleniia massy perevozimogo gruza musorovoznym transporttom [Choice of rational method for determining the weight of cargo trucks for garbage collection]. Vestnik Permskogo gosudarstvennogo tekhnicheskogo universiteta. Okhrana okruzhaiushchei sredy, transport, bezopasnost' zhiznedeiatel'nosti, 2011, no. 1, pp. 119-123.

4. Kopotilov V.I. Avtomobili: Teoreticheskie osnovy [Cars: Theoretical Foundations]. Tiumenskii gosudarstvennyi neftegazovyi universitet, 1999. $403 \mathrm{p}$.

5. Musorovoz MK-20. Rukovodstvo po ekspluatatsii [Garbage MK-20. Operation Manual]. OAO “Saranskii zavod avtosamosvalov", 2008. 55 p. 
6. Kuznetsov V.M. Kommunal'naia tekhnika 2001 [Grounds Care 2001]. Moscow: RIA “Rossbiznes", 2001. 176 p.

7. Sister V.G. [at al.] Tverdye bytovye otkhody (sbor, transport i obezvrezhivanie) [Municipal solid waste (collection, transportation and disposal)]. Moscow: Akademiia kommunal'nogo khoziaistva imeni K.D. Pamfilova, 2001. 319 p.

8. Kontseptsiia obrashcheniia $\mathrm{s}$ tverdymi bytovymi otkhodami $\mathrm{v}$ Rossiiskoi Federatsii [The concept of solid waste management in the Russian Federation]. Chistyi gorod, 2003, no. 2(22), pp. 36-48.

9. Karbanovich I.I. Ekonomiia avtomobil'nogo topliva: opyt i problem [Automotive fuel economy: experience and problems]. Moscow: Transport, 1992. $145 \mathrm{p}$.

10. Erokhov V.I. Ekonomichnaia ekspluatatsiia avtomobilia [Car economic exploitation]. Moscow: DOSAAF, 1986. 126 p.

11. Lavrikov I.N., Pen'shin N.V. Ekonomika avtomobil'nogo transporta: uchebnoe posobie [The economy of road transport: a tutorial]. Tambovskii gosudarstvennyi tekhnicheskii universitet, 2011. $116 \mathrm{p}$.

12. Sichinava G.A. Otsenka ekonomicheskoi effektivnosti investitsionnykh proektov v stroitel'stve: metod. ukazaniia $\mathrm{k}$ ekonomicheskoi chasti diplomnykh proektov [Estimation of economic efficiency of investment projects in building: guidelines for the economic part of the graduation projects]. Ukhtinskii gosudarstvennyi tekhnicheskii universitet, $2010.98 \mathrm{p}$.

13. Turevskii I.S. Ekonomika i upravlenie avtotransportnym predpriiatiem [Economics and management of road transport enterprise]. Moscow: Vysshaia shkola, 2005. $222 \mathrm{p}$.

14. Glovatskii L.V., Gasanov P.G., Vasil'ev S.V. Upravlenie otkhodami: vchera, segodnia, zavtra [Waste Management: yesterday, today and tomorrow]. Spravochno-informatsionnaia sistema "Otkhody.ru”, 2008, available at: www.waste.ru/modules/section/item.php?itemid=15 (accessed 26 January 2016).

15. Sadokhina E.L. Programmnoe obespechenie v ekologii. Sushchestvuiushchee polozhenie i perspektiva [The software in the environment. Current situation and outlook]. Spravochno-informatsionnaia sistema "Otkhody.ru", 2006, available at: www.waste.ru/modules/ section/item.php?itemid=63 (accessed 26 January 2016).

Получено 17.02.2016 


\section{Об авторах}

Лобов Николай Владимирович (Пермь, Россия) - доктор технических наук, профессор кафедры «Автомобили и технологические машины» Пермского национального исследовательского политехнического университета (614990, г. Пермь, Комсомольский пр., 29, e-mail: lobov@pstu.ru).

Пестриков Сергей Анатольевич (Пермь, Россия) - кандидат экономических наук, доцент кафедры «Автомобили и технологические машины» Пермского национального исследовательского политехнического университета (614990, г. Пермь, Комсомольский пр., 29, e-mail: pestrikovs@mail.ru).

Монченко Сергей Александрович (Пермь, Россия) - магистрант кафедры «Автомобили и технологические машины» Пермского национального исследовательского политехнического университета (614990, г. Пермь, Комсомольский пр., 29, e-mail: s.a.monchenko@gmail.com).

\section{About the authors}

Nikolai V. Lobov (Perm, Russian Federation) - Doctor of Technical Sciences, Professor, Department of Automobiles and Technological Machines, Perm National Research Polytechnic University (29, Komsomolsky av., Perm, 614990, Russian Federation, e-mail: lobov@pstu.ru).

Sergei A. Pestrikov (Perm, Russian Federation) - Ph.D. in Economical Sciences, Associate Professor, Department of Automobiles and Technological Machines, Perm National Research Polytechnic University (29, Komsomolsky av., Perm, 614990, Russian Federation, e-mail: pestrikovs@mail.ru).

Sergei A. Monchenko (Perm, Russian Federation) - Master Student, Department of Automobiles and Technological Machines, Perm National Research Polytechnic University (29, Komsomolsky av., Perm, 614990, Russian Federation, e-mail: s.a.monchenko@gmail.com). 